\title{
Lobster (Panulirus interruptus) Pyloric Muscles Express the Motor Patterns of Three Neural Networks, Only One of Which Innervates the Muscles
}

\author{
Jeff B. Thuma, ${ }^{1 \star}$ Lee G. Morris, ${ }^{2 \star}$ Adam L. Weaver, ${ }^{2}$ and Scott L. Hooper ${ }^{1}$ \\ ${ }^{1}$ Neuroscience Program, Department of Biological Sciences, Ohio University, Athens, Ohio 45701, and 2Department of Biology, Emory University, Atlanta, \\ Georgia 30322
}

\begin{abstract}
In several systems, including some well studied invertebrate "model" preparations, rapid, rhythmic inputs drive slow muscles. In this situation muscle contractions can summate temporally between motor neuron bursts, tonically contract, and low-pass filter broad-band input. We have investigated how the muscles innervated by each motor neuron type of the rapid, rhythmic (cycle period, $\sim 1$ sec) lobster pyloric network respond when driven by previously recorded in vitro pyloric network activity from intact stomatogastric nervous systems. Under these conditions the much slower gastric mill and cardiac sac networks of the stomatogastric nervous system are also active and modify pyloric activity. All of the muscles show pyloric timed phasic contractions that ride on a sustained tonic contraction; muscle activity can range from being almost completely phasic to almost completely tonic. The modifications of pyloric neuron activity induced by gastric mill (cycle period, $\sim 10 \mathrm{sec}$ ) activity result in some pyloric muscles showing prominent, gastric mill-timed, changes in either phasic or tonic contraction amplitude. The strong modification of pyloric neuron activity induced by cardiac sac (cycle period, $\sim 60$ sec) activity alters the contractions of all pyloric muscles. These changes are sufficient that for some muscles, in some preparations, the primary muscle output is cardiac sac-timed. This is the first work to examine the motor responses of all pyloric muscle classes to spontaneous stomatogastric activity and shows that the pyloric motor pattern is a complex combination of the activities of three neural networks, although only one (the pyloric) innervates the muscles.
\end{abstract}

Keywords: pyloric network; lobster; stomatogastric; motor pattern; muscle; invertebrate; central pattern generator; Panulirus interruptus

\section{Introduction}

A fundamental goal of neuroscience is explaining the neural basis of behavior. Great progress has been made in understanding the neural networks that produce rhythmic motor patterns, particularly in invertebrate model preparations (Marder and Calabrese, 1996). Less investigated is the transformation of neural activity by muscle into movement, particularly in the preparations best understood on the neural level. This lack is a concern because many muscles in these preparations contract and relax slowly compared with the cycle periods of their neural input (Atwood, 1973; Mason and Kristan, 1982; Hetherington and Lombard, 1983; Carrier, 1989; Hall and Lloyd, 1990; McPherson and Blankenship, 1992; Ellis et al., 1996; Koehnle et al., 1997; Harness, 1998; Harness et al., 1998; Morris and Hooper, 1998; Morris et al., 2000). In this case the muscle contractions can summate temporally between motor neuron bursts, tonically contract, and low-pass filter their input (Morris and Hooper, 1998; Morris et al., 2000). Another

\footnotetext{
Received June 13, 2003; revised Aug. 7, 2003; accepted Aug. 11, 2003.

This research was supported by grants to S.L.H. from the National Science Foundation, the Human Frontier Science Program, National Institutes of Health, and Ohio University and its research council. We thank R. A. DiCaprio for discussion and advice.

*J.B.T. and L.G.M. contributed equally to this work.

Correspondence should be addressed to S. L. Hooper, Neuroscience Program, Department of Biological Sciences, Irvine Hall, Ohio University, Athens, OH 45701. E-mail: hooper@ohio.edu.

Copyright $\odot 2003$ Society for Neuroscience $\quad$ 0270-6474/03/238911-10\$15.00/0
}

concern is that many of these muscles facilitate strongly, and small changes in motor neuron burst spike number thus could induce large changes in muscle contraction amplitude.

To address these issues, we have been investigating the contraction properties of the pyloric muscles of the lobster (Panulirus interruptus) stomatogastric system. We have shown that many pyloric muscles are slow, that their response to artificial, pyloric-like rhythmic input is a tonic contraction on which phasic, pyloric timed contractions ride, and that many of them extract low-frequency modifications of pyloric motor neuron activity imposed by other (gastric mill, cardiac sac) stomatogastric neural networks (Ellis et al., 1996; Koehnle et al., 1997; Harness, 1998; Harness et al., 1998; Morris and Hooper, 1998; Morris et al., 2000). We also have quantified the changes in pyloric network output that these other networks induce (Thuma and Hooper, 2002, 2003). However, the pyloric motor pattern, when driven by actual pyloric neural activity with gastric mill and cardiac sac modification present, has not been described.

The pyloric network has five motor neuron types, some of which innervate multiple muscles (Maynard and Dando, 1974). We here describe the contractions of a set of pyloric muscles that includes muscles innervated by each pyloric motor neuron type. Each muscle shows a mixture of pyloric and cardiac sac-timed, or pyloric, gastric mill, and cardiac sac-timed, activity. The pyloric motor pattern is thus a complex combination of the activities of 


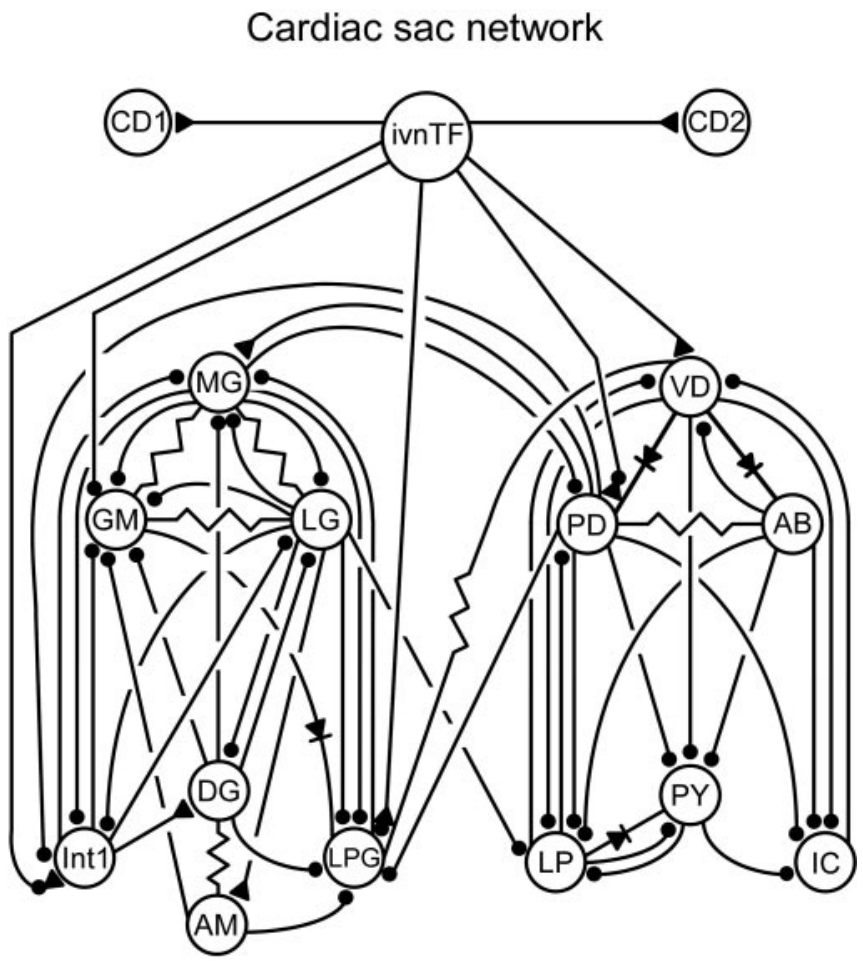

Gastric mill network

Figure 1. Synaptic connectivity diagram for the cardiac sac, gastric mill, and pyloric networks. Ball and stick connections represent ionotropic inhibitory synapses; triangle and stick connections, ionotropic excitatory synapses; resistors, electrical coupling; diodes, rectifying electrical coupling. The ivnTF fibers inhibit the GM neurons, excite the VD neuron, and make combined inhibitory/excitatory synapses onto the PD and LPG neurons and interneuron 1. The $L G$ neuron inhibits the LP neuron. The VD neuron is coupled electrically to the LPG neurons, and the PD neurons inhibit the LPG neurons. CD1, CD2, Cardiac sac dilator neurons 1 and 2; ivnTF, inferior ventricular nerve through fibers; $M G$, median gastric; $G M$, gastric mill; $L G$, lateral gastric; DG, dorsal gastric; LPG, lateral posterior gastric; AM, anterior median; Int1, interneuron 1.

three neural networks, although only one (the pyloric) innervates the muscles.

Some of these data have been published previously in abstract form (Morris et al., 1999).

\section{Materials and Methods}

Lobsters were obtained from Don Tomlinson Commercial Fishing (San Diego, CA) and maintained at $10-15^{\circ} \mathrm{C}$ in aquaria with circulating artificial sea water. Two types of experiments were performed. The first were $600 \mathrm{sec}$ recordings of pyloric neuron activity with the stomatogastric nerve intact. Stomachs were dissected in the standard manner (Selverston et al., 1976). Preparations were superfused continuously (15-30 $\mathrm{ml} / \mathrm{min}$ ) with $12-14^{\circ} \mathrm{C}$ Panulirus saline. Extracellular nerve recordings were made with stainless steel pin electrodes and an A-M Systems (Everett, WA) amplifier. Intracellular neuronal recordings were made with glass microelectrodes (filled with $0.55 \mathrm{M} \mathrm{K}_{2} \mathrm{SO}_{4}$ and $0.02 \mathrm{M} \mathrm{KCl}$; resistance, $10-20 \mathrm{M} \Omega$ ) and an Axoclamp 2A or 2B (Axon Instruments, Foster City, CA). Data were displayed on a Tektronix (Beaverton, OR) oscilloscope, recorded on a MicroData Instruments (Woodhaven, NY) DT-800 digital data recorder, and transferred to computer with a Cambridge Electronics Design (CED, Cambridge, UK) 1401 Plus and Spike 2 software (CED).

Figure 1 shows the synaptic connectivities of the pyloric, gastric mill, and cardiac sac networks and their known interconnections. Pyloric activity was monitored by intracellular recording from neuron somata or extracellular recording from appropriate motor nerves: lateral pyloric (LP) neuron, lateral ventricular nerve (lvn); ventricular dilator (VD) and

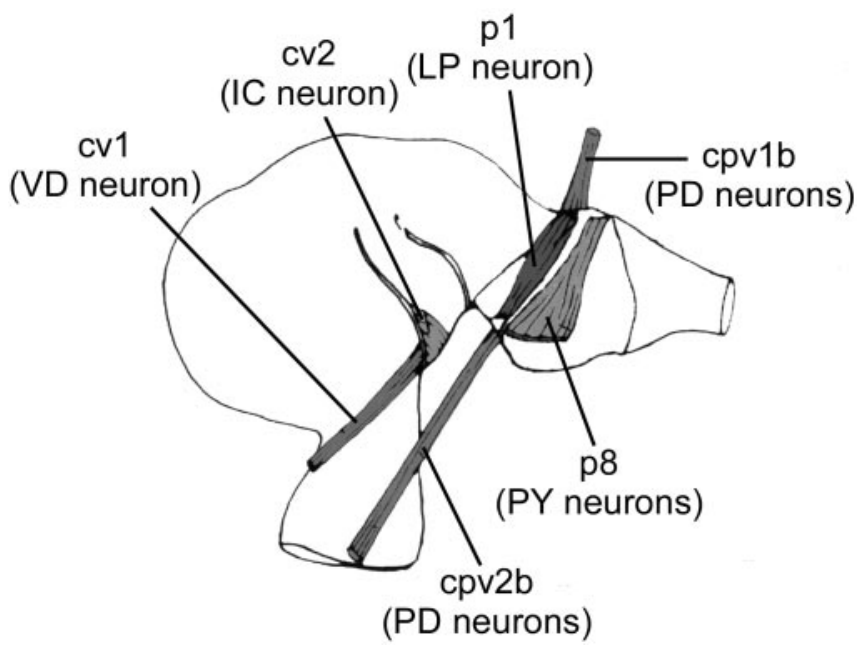

Figure 2. Muscle locations. cpv1b and cpv2b are innervated by the two PD neurons, cv1 by the VD neuron, $\mathrm{CV} 2$ by the IC neuron, $\mathrm{p} 1$ by the LP neuron, and $\mathrm{p} 8$ by six to eight $\mathrm{PY}$ neurons.

inferior cardiac (IC) neurons, and median ventricular nerve (mvn). Pyloric dilator (PD) neurons and pyloric (PY) neuron activity were always recorded intracellularly because there are two PD and six to eight PY neurons, and individual PD and PY neuron activity cannot be distinguished in extracellular recordings. Gastric mill activity (GM neurons) was monitored with extracellular anterior lateral (aln) or gastropyloric nerve recordings. The VD neuron (mvn) was used as a marker for cardiac sac activity (Thuma and Hooper, 2003).

In the second set of experiments the pyloric muscle response to the neural activity recorded in the first set of experiments was observed. Stomachs were dissected by using standard techniques for measurements of muscle contraction (Maynard and Dando, 1974; Selverston et al., 1976; Morris and Hooper, 1997, 1998; Morris et al., 2000), taking great care not to stretch the muscles or expose them to gastric juices [pyloric muscles 1 and 8 (p1 and p8), ventral cardiac muscles 1 and 2 (cv1 and cv2), cardiopyloric valve muscles $1 \mathrm{~b}$ and $2 \mathrm{~b}$ (cpv1b and cpv2b); Fig. 2]. The preparation was superfused continuously (15-30 $\mathrm{ml} / \mathrm{min}$ ) with $12-$ $14^{\circ} \mathrm{C}$ oxygenated Panulirus saline with $40 \mathrm{~mm}$ dextrose. Contractions were measured by connecting one end of the arm of a Harvard Apparatus (Holliston, MA) 60-3000 isotonic transducer to a wire hooked through chitin at the origin of the muscle. Muscle rest length was maintained at approximately physiological levels (determined from in situ observations). Muscle loading was determined by inducing single contractions and adjusting muscle load to achieve consistent maximum contraction amplitude with full relaxation after the stimulation. A support bar then was placed under the other end of the transducer arm to prevent muscle overstretching.

Stimulation (100 $\mu \mathrm{sec}$ shocks) was done with a polyethylene suction electrode on the mvn or lvn, with the nerve cut above the electrode to prevent spontaneous pyloric network input from reaching the muscle. There are two PD axons and six to eight PY axons in the lvn. So that activation of all axons innervating a muscle could be ensured, stimulation amplitude was increased incrementally by using single burst stimulations until contraction amplitude ceased increasing; stimulation with this amplitude causes only a single action potential per shock (Hoover et al., 2002). The nerves were stimulated with a WPI Pulsemaster A300 stimulator (World Precision Instruments, Sarasota, FL) driven by the CED, using the previously recorded motor neuron activity and a script written in Spike 2 (e.g., the VD neuron recording was used to stimulate the mvn and hence cv1). Muscle response was recorded on tape and transferred to computer by using the CED 1401. The data shown here are from 22 muscle experiments.

\section{Results}

The pyloric neuromuscular system is driven by the pyloric neural network, which has a cycle period of $\sim 1 \mathrm{sec}$ and fires $100-500$ 


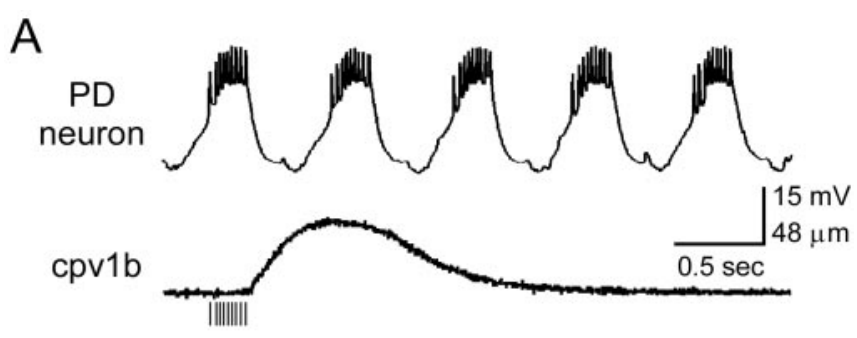

B

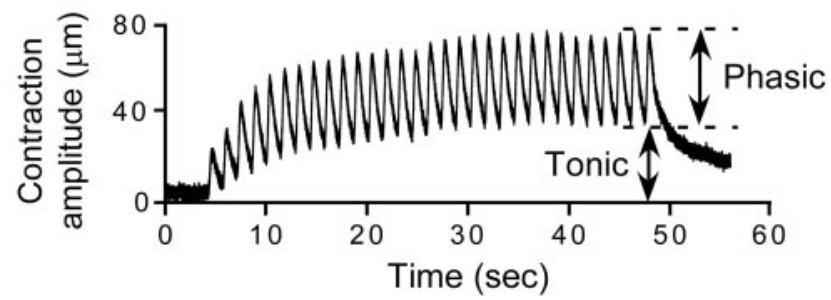

Figure 3. Temporal summation in the muscles. $A$, When stimulated with a train of spikes (vertical lines under muscle trace) mimicking real neural input, the cpv1b muscle relaxes too slowly to relax fully before the next burst of spikes would be received. $B$, Because of this, when stimulated with rhythmic spike bursts, the muscle contractions temporally summate, creating a sustained contraction (tonic component) on top of which ride phasic contractions corresponding to each spike burst.

msec duration bursts of action potentials. Pyloric muscles are nonspiking muscles that contract as a graded function of their neural input. Based on the bursting nature of the network, it was long assumed that the pyloric motor pattern was completely rhythmic (i.e., that the muscle contractions each motor neuron burst produced fully relaxed between bursts). Work on pyloric muscle contractions evoked by nerve stimulation in the crab Cancer borealis and shrimp Palaemon serratus supported this assumption (Meyrand and Moulins, 1986; Meyrand and Marder, 1991; Jorge-Rivera and Marder, 1996).

However, in Panulirus some pyloric muscles relax too slowly to follow faithfully their motor neuron bursts (Morris and Hooper, 1997). The top trace of Figure $3 A$ shows the characteristic rhythmic bursting of a PD neuron. The bottom trace shows an isotonic contraction of muscle cpv1b, an extremely slow muscle innervated by the PD neurons. The contraction was produced by stimulating the motor nerve with the same spike number and average spike frequency as the first burst in the neuron trace. If the neuron actually were driving the muscle, the next burst would arrive before the muscle had relaxed completely. When the motor nerve was stimulated rhythmically by using physiologically relevant parameters, the muscle contractions temporally summated (Fig. $3 B$ ). Once the contractions stabilized, they consisted of a sustained, tonic baseline contraction on top of which rode phasic contractions matching the rhythmic motor nerve stimulations.

The stomatogastric nervous system also contains the much more slowly cycling gastric mill and cardiac sac networks (cycle periods, 5-10 and $\sim 60 \mathrm{sec}$, respectively). Gastric mill and cardiac sac activity modify pyloric activity (Selverston et al., 1976; Mulloney, 1977; Russell and Hartline, 1981; Sigvardt and Mulloney, 1982; Claiborne and Selverston, 1984). In particular, the overall spike frequency (burst spike number divided by cycle period) of all pyloric neurons varies with gastric mill and cardiac sac activity (Thuma and Hooper, 2002, 2003). Phasic and tonic contraction amplitudes strongly depend on overall spike frequency (Morris, 1998 ) because (1) spike number mainly determines phasic contraction amplitude and (2) the temporal summation that gives rise to the tonic contraction depends strongly on phasic contraction amplitude and the duration of the interburst interval during which the muscle relaxes. If overall spike frequency is changed consistently for several cycles, phasic and tonic contraction amplitudes and their ratio thus also change.

The modifications of pyloric activity that gastric mill and cardiac sac activity induce last for several pyloric cycles (Thuma and Hooper, 2002, 2003) and thus alter phasic and tonic contraction amplitude. Although some of these changes have been described for a few pyloric muscles (Morris et al., 2000), their effects on all pyloric muscle classes have not. In our neural experiments we have preparations in which only the pyloric network was active, in which the pyloric and gastric mill networks, but not the cardiac sac, were active, and in which all three networks were active. Also, in several preparations with cardiac sac activity the cardiac sac bursts occurred with a sufficiently long cycle period that pyloric and gastric mill activity could be observed without cardiac sac interference during the cardiac sac interburst intervals. However, we have never observed cardiac sac activity without the gastric mill also being active. Therefore, we show here the activity of representatives of all pyloric muscle classes to pyloric activity alone, pyloric activity modified by gastric mill activity, and pyloric activity modified by gastric mill and cardiac sac activity.

\section{Pyloric network activity alone}

Figure 4 shows the length change of each pyloric muscle to stimulation with the activity of its innervating neuron when gastric mill and cardiac sac activity was not altering pyloric network activity. All muscles shown in this figure came from a single preparation and all neuron recordings from a single experiment. The horizontal line under each muscle trace shows muscle rest length; the vertical lines joining this line to the muscle trace are shown to accentuate that the muscle contraction consists of a phasic and a tonic component. The small vertical lines on the bottom horizontal line in each panel indicate motor neuron spikes. In this preparation p1 (LP neuron innervated), cv1 (VD neuron innervated), p8 (PY neuron innervated), and cpv2b (PD neuron innervated) relaxed fairly quickly; approximately one-half of their contraction amplitude was a sustained tonic contraction, whereas the other one-half was phasic contractions matching the rhythmic activity of their pyloric neuron. cv2 (IC neuron innervated) relaxed more quickly than these muscles and showed very little tonic contraction. cpvlb (PD neuron innervated) relaxed very slowly and showed an almost completely tonic contraction.

Tonic contraction depends on cycle period and motor neuron burst duration (Morris and Hooper, 1998) (also see Discussion and Fig. 11). For most muscles the tonic contraction amplitude therefore varied from preparation to preparation depending on network cycle period, motor neuron burst duration, and preparation-specific differences in muscle relaxation rate. [A notable exception was cpvib (PD), which relaxed so slowly that it produced almost exclusively tonic contractions in all experiments.] Importantly, however, within a preparation the phasic and tonic contraction amplitudes do not show slow rhythmic changes in the absence of gastric mill or cardiac sac activity (Morris and Hooper, 1998, 2001).

\section{Pyloric network activity modified by the gastric mill}

Gastric mill activity strongly alters IC and PY neuron overall spike frequency (OSF), less strongly alters PD and LP neuron OSF, and only very weakly alters VD neuron OSF (Thuma and Hooper, 2002). These changes are reflected by corresponding gastric mill-timed changes in phasic and tonic contraction am- 


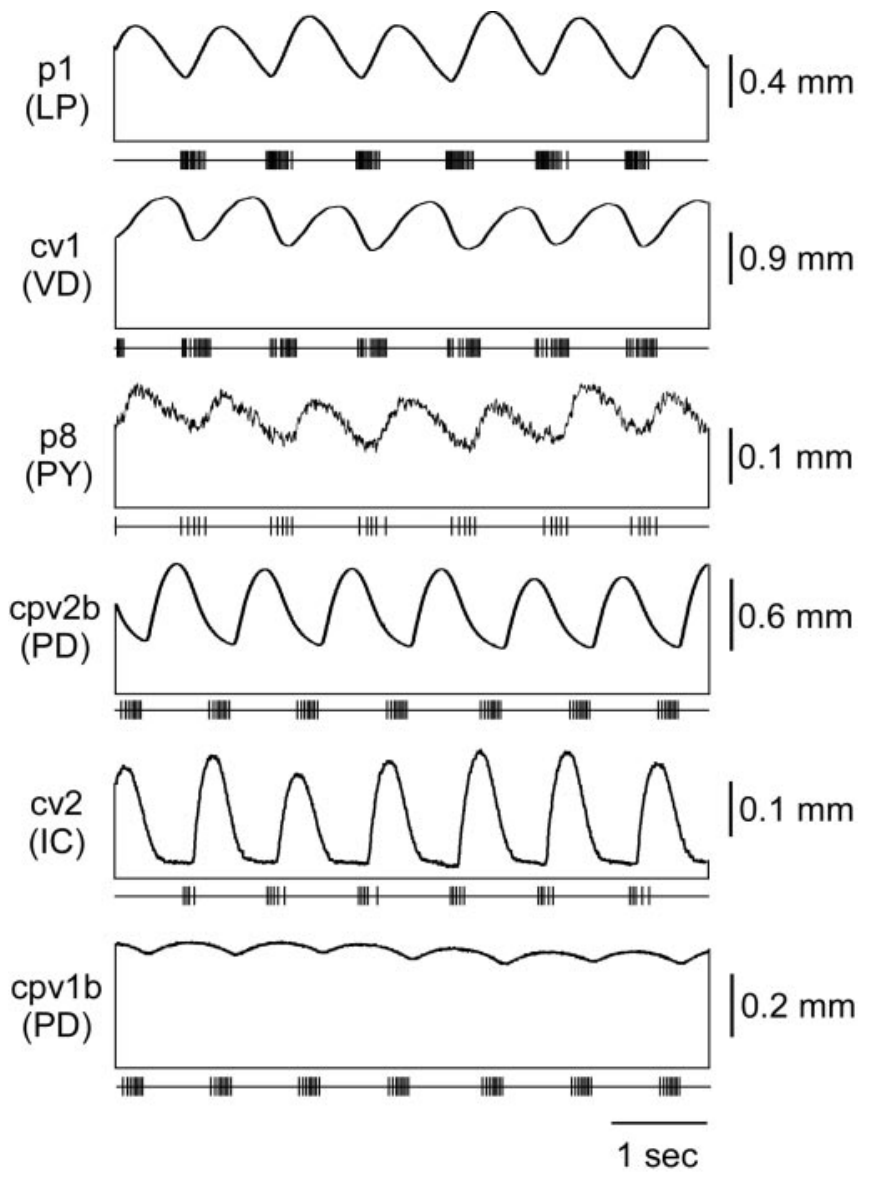

Figure 4. Muscle responses when the gastric mill and cardiac sac are not modifying pyloric network activity. The LP (p1), PY (p8), VD (cv1), and one PD (cpv2b) neuron-innervated muscle relax relatively quickly, and only approximately one-half of their total contraction amplitude is tonic. The IC neuron-innervated muscle (cv2) relaxes quickly, and its contractions are almost entirely phasic. The other PD neuron-innervated muscle (cpv1b) relaxes extremely slowly, and its contraction is almost completely tonic. In this and Figures 5-10 the horizontal line under each muscle trace shows muscle rest length; the vertical lines joining this line to the muscle trace are placed to accentuate that the muscle contraction consists of a phasic and a tonic component. The small vertical lines on the bottom horizontal line in each panel indicate motor neuron spikes.

plitude in the muscles these neurons innervate (Fig. 5) despite the fact that no gastric mill neuron innervates any pyloric muscle. The muscle contractions in the figure were taken from different preparations, but the neural input used to stimulate the muscles came from a single experiment. The dashed lines show one gastric mill cycle (aln trace). The horizontal lines connected to each muscle trace by vertical lines again show full relaxation of that muscle (note again the large tonic component of the contractions), and the traces below represent motor neuron firing. $\mathrm{p} 1$ (LP), cv2 (IC), p8 (PY), and cpv2b (PD) show gastric mill-timed variations in phasic and tonic contraction amplitude. cv1 (VD) and cpvib (PD) show very little or no gastric mill-timed tonic contraction variation.

\section{Pyloric network activity modified by the gastric mill and cardiac sac}

Cardiac sac activity strongly alters OSF of all pyloric neurons (Thuma and Hooper, 2003). For the VD neuron this effect is a simple increase in OSF. For the LP neuron it is a simple decrease in OSF. PY neurons can show an increase or a decrease. The PD and IC neurons show more complex responses. PD neuron over-

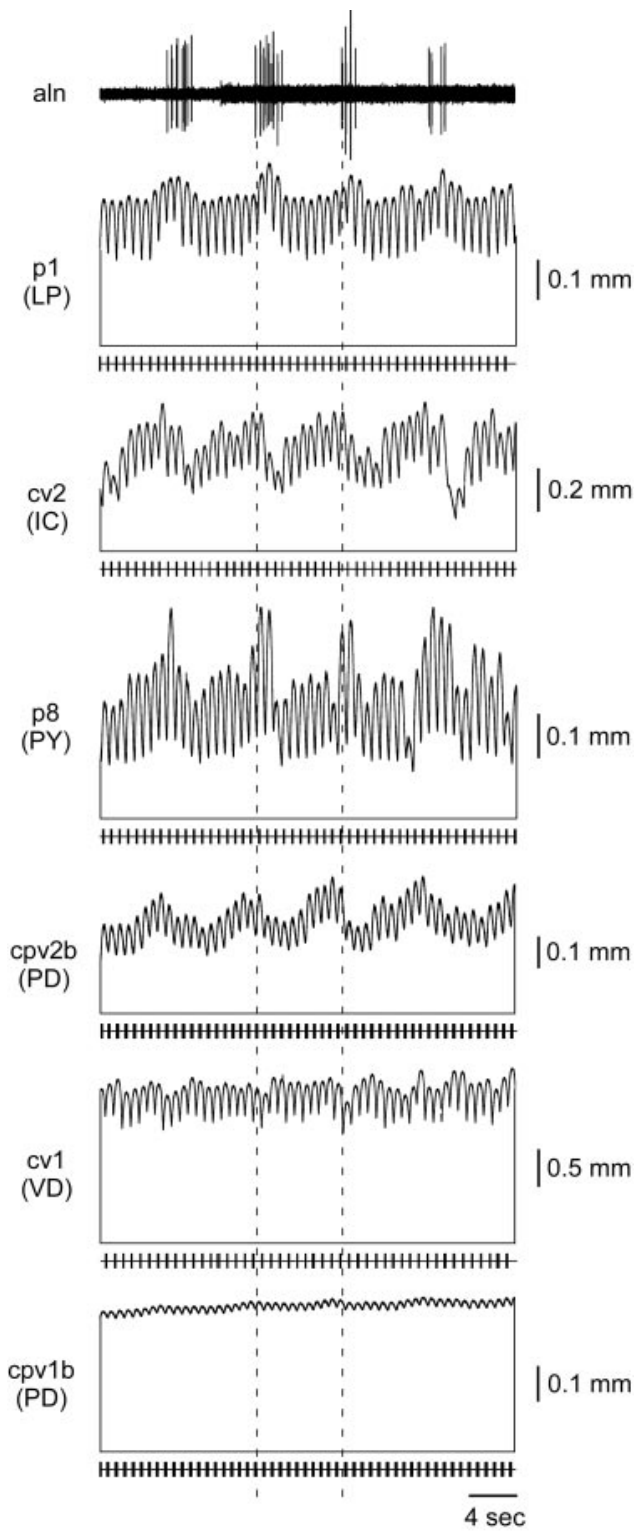

Figure 5. Muscle responses when the gastric mill (aln) is active. The phasic and tonic contraction amplitudes of the LP (p1), IC (cv2), PY (p8), and one PD (cpv2b) neuron-innervated muscles vary in gastric mill time (dashed lines mark one gastric mill cycle period). The VD (cv1) and other PD neuron-innervated muscle (cpv1b) are affected little by gastric mill activity.

all spike frequency increases just before a cardiac sac network burst, decreases during it, and increases afterward. IC neuron overall spike frequency decreases during the cardiac sac bursts and then shows a sustained increase in activity. The muscle responses primarily mirror these changes in neuron firing (Figs. 6-8).

Figure 6 shows p1, cpv1b, and cpv2b muscle responses when driven with actual neural input (recorded from the LP neuron for the $\mathrm{p} 1$ muscle and recorded from a PD neuron for muscle cpv1b and cpv2b). The neural recordings are from a single experiment, but the muscle traces are from different preparations. Before the cardiac sac burst, p1 showed a large tonic contraction with small, pyloric timed phasic contractions and some gastric mill-timed activity (inset). During the cardiac sac burst (black rectangles at top) the LP neuron is inhibited, which caused p1 to relax. Depending on cardiac sac burst length, in some preparations $\mathrm{p} 1$ fully 


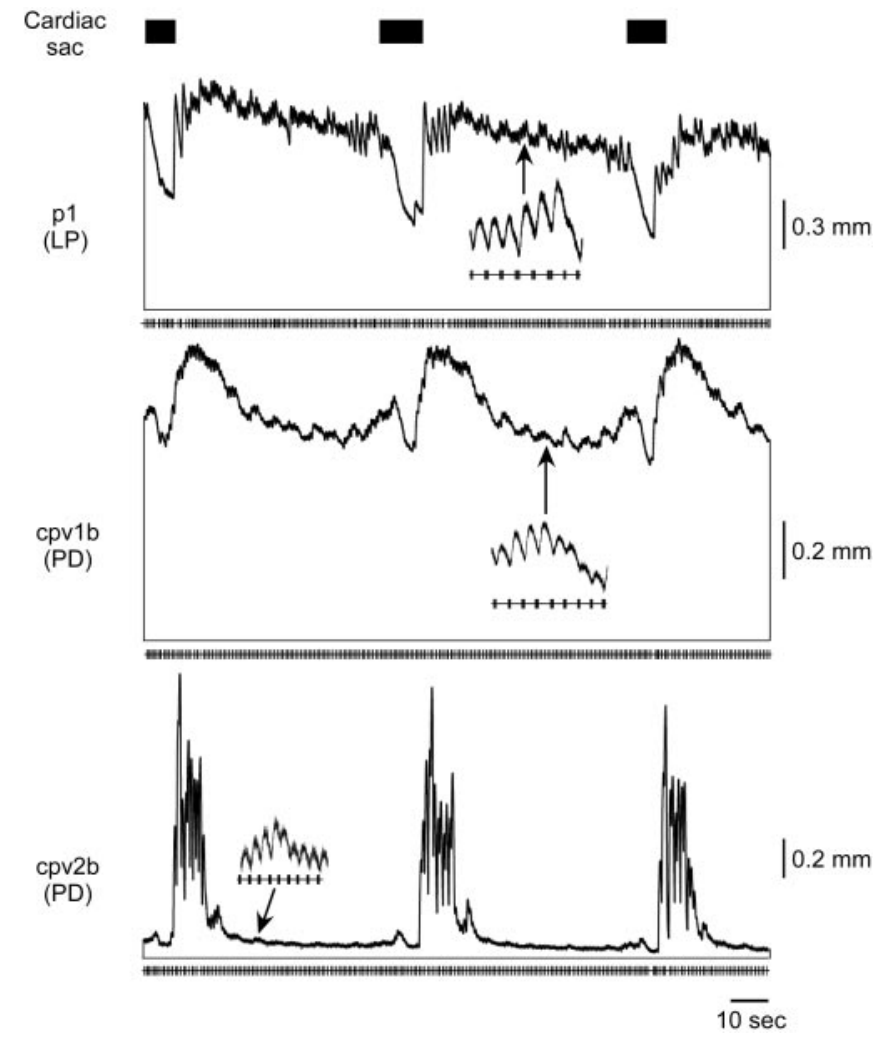

Figure 6. $\quad \mathrm{p} 1(\mathrm{LP}), \mathrm{cpv} 1 \mathrm{~b}(\mathrm{PD})$, and $\mathrm{cpv} 2 \mathrm{~b}(\mathrm{PD})$ response when the cardiac sac (black rectangles) and gastric mill are active. The LP neuron is inhibited during cardiac sac activity; thus p1 shows a large relaxation during that time. Between cardiac sac bursts small gastric mill-timed variations are present (insets). cpv1b (PD) and cpv2b (PD) contractions show an increase just before, a decrease during, and a large sustained increase after cardiac sac activity.

relaxed before the pyloric rhythm resumed, and the muscle returned to activity similar to that before the cardiac sac burst.

cpvib and cpv2b are both innervated by the PD neurons, but their muscle properties are very different (Morris and Hooper, 1997). cpv1b is a very slow muscle. Before the cardiac sac burst, its contraction was a sustained, tonic contraction with very small phasic contractions and moderate gastric mill-timed activity (inset). The response of the muscle to cardiac sac activity almost exactly mirrors the cardiac sac-induced changes in PD neuron activity (Thuma and Hooper, 2003): an increase-decrease-increase change in tonic contraction amplitude. cpv2b is a much faster contracting and relaxing muscle than cpvib and shows extremely large facilitation (Morris and Hooper, 1996). Its phasic contraction amplitude is therefore extremely sensitive to PD neuron burst spike number and interburst interval. PD neuron spike number before cardiac sac activity is usually too small to produce large contractions in this muscle; consequently, its phasic and tonic contractions, and their gastric mill-timed modifications, are usually small between cardiac sac bursts. The increase in PD neuron spike number after the cardiac sac burst, however, can result in cpv2b contractions $>50$ times the size of the pyloric contractions before the burst. This extremely large increase in contraction amplitude can last for tens of seconds; as a result, the contractions of the muscle can (as here) be limited to occurring only after each cardiac sac burst.

Figure 7 shows the response of cv1 (VD) and cv2 (IC) to cardiac sac activity. The neural recordings used to stimulate the muscles are from the same experiment, but the muscles are from different preparations. In this preparation the tonic contraction of cv1 was approximately five times larger than the phasic contractions produced by each VD neuron burst. The muscle showed very little gastric mill modulation (inset). During cardiac sac activity (black rectangles) the muscle produced a large contraction. After the cardiac sac burst, the tonic contraction of the muscle decreased before returning to the level before cardiac sac activity. The IC neuron is inhibited during cardiac sac activity and thus cv2 relaxed during this time. For 20-30 sec after cardiac sac activity the cv2 phasic contraction amplitude was increased before returning to the gastropyloric rhythm (inset) it expressed before cardiac sac activity.

Cardiac sac activity can excite or inhibit the PY neurons (Thuma and Hooper, 2003). The effect of cardiac sac activity on PY neuron-innervated muscles thus depended on the response of the neuron being used in the stimulation. Figure $8 \mathrm{~A}$ shows the response of $\mathrm{p} 8$ when stimulated by a PY neuron excited during, and Figure $8 B$ by one inhibited during, cardiac sac activity. The excited PY neuron produced a very large contraction toward the end of cardiac sac activity. This contraction was 20-25 times larger than the phasic pyloric contractions and 5-10 times larger than the gastric mill-timed variation in contraction amplitude (inset). Stimulation with the inhibited PY neuron, alternatively, caused almost full muscle relaxation during the cardiac sac burst, followed by a return to the gastropyloric activity present before the cardiac sac burst.

Figure 9 summarizes the activity of each pyloric muscle type when driven by pyloric activity modified by both gastric mill and cardiac sac activity. The muscles were from different preparations, but the neural recordings were from a single preparation. The dashed lines show cardiac sac (mvn) burst duration and gastric mill (aln) cycle period. cv1 (VD), cpv1b (PD), and p1 (LP) showed a large tonic contraction that persisted throughout gastric mill and cardiac sac activity. cv1 showed no gastric mill-timed variation, an increased contraction during the cardiac sac burst, and a sustained decrease after the burst. cpv1b (PD) showed very small gastric mill variations, a decreased contraction during the cardiac sac burst, and a sustained increase after it. In this experiment 1 showed relatively small gastric mill-timed variations, a decreased contraction during the cardiac sac burst, and a large contraction immediately after. cpv2b (PD) showed pronounced gastric mill variation, with peak contraction amplitude occurring at the beginning of each GM neuron burst. During the cardiac sac burst the muscle fully relaxed and showed very large pyloric timed contractions after the burst. cv2 showed large gastric mill variations, with the peak contraction amplitude occurring just before each GM neuron burst. The muscle almost fully relaxed during the cardiac sac burst and showed a large contraction after the burst. p8 showed large gastric mill-timed variations, with the peak contraction amplitude occurring during the GM neuron burst; in this preparation the muscle fully relaxed each gastric mill cycle. This experiment used a PY neuron excited during cardiac sac activity, and thus the muscle shows a large cardiac sac-timed contraction.

Although the data presented in Figure 9 are representative, they are not averaged. The reason for this is the muscle and neural variability across preparations. The left column in Figure 10 shows the responses of all of the muscles in our database when driven by data from the same segment of a single neural experiment. The top trace shows extracellular activity from the mvn, which contains the VD (large spikes) and IC neuron (small spikes) axons. A cardiac sac burst occurred in the middle of the segment that is shown. The second trace shows extracellular gastric mill activity: six gastric mill cycles split into two groups of 
three by the intervening cardiac sac burst. Four cvl muscles (different preparations are shown in different color traces) were driven by the VD neuron data shown in trace 1. Although all four muscles had qualitatively similar contraction activity (very little gastric mill-timed variation and a sustained, nonrhythmic contraction during the cardiac sac bursts), their activities showed large quantitative differences. For instance, the muscles represented by green and blue had very different tonic contraction amplitudes, the muscles represented by black and red had much larger pyloric-timed phasic contraction amplitudes than the green and blue traces, and the blue, red, and green traces showed an increase in tonic amplitude during the cardiac sac burst, but the black trace did not.

Qualitative similarity but quantitative difference was also apparent when five cv2 muscles were driven by using the IC neuron firing data shown in trace 1. All five muscles showed gastric mill-timed variations in activity, decreased contraction amplitudes during the cardiac sac burst, and dramatically increased contraction amplitudes after the burst. However, the muscle represented by black shows much larger phasic contractions and a larger tonic contraction than did the other muscles. Comparison of the other muscles [cpvib (PD), 3 muscles; cpv2b (PD), 3 muscles; p1 (LP), 5 muscles; p8 (driven by a PY neuron inhibited by cardiac sac activity), 5 muscles] shows that in each case, although all muscles of each type showed qualitatively similar activity, their responses were quantitatively different.

It is possible that, despite these differences, some sort of averaged response could have been calculated except that this difference between muscle responses when driven by identical input is coupled with the neural activity in different preparations having similarly great variation. The right column of Figure 10 shows mvn and gastric mill extracellular recordings (top two traces in each panel), and the activity of the same p8 (PY) muscle, for a segment of three experiments that in all cases included a cardiac sac burst. Comparison of the muscle activity in the three panels shows that again the contractions of the muscle were qualitatively the same; in all three cases gastric mill-timed variations were present, and p8 contraction decreased during the cardiac sac burst (in all three experiments the PY neuron recorded from was inhibited during cardiac sac activity). However, the PY neurons present in the three neural experiments had sufficiently different activities that the contractions of the muscle were quantitatively very different when driven by them. For instance, with the PY neuron activity present in the top and bottom preparations the muscle showed very little tonic contraction, whereas in the middle preparation almost one-half

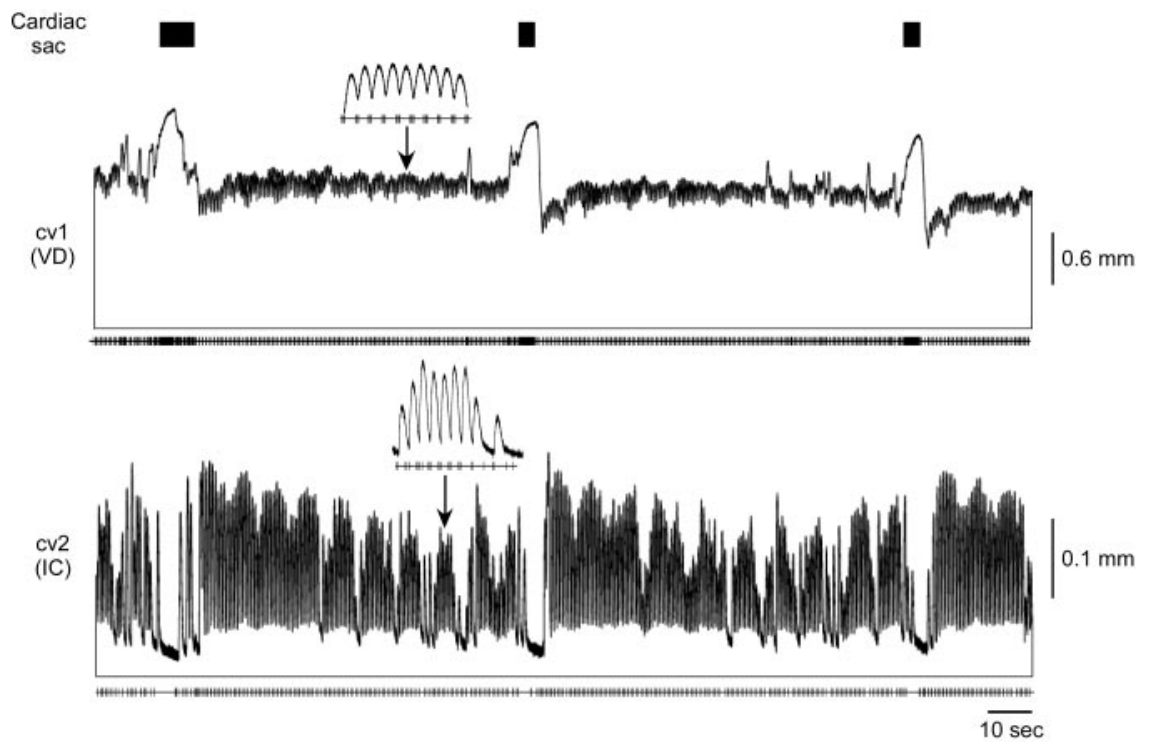

Figure 7. $\quad$ cv1 (VD) and cv2 (IC) response when cardiac sac (black rectangles) and gastric mill are active. cv1 shows a large increase in contraction amplitude during cardiac sac activity, followed by a decrease below and gradual return to the tonic contraction amplitude present before cardiac sac activity. Very little gastric-timed amplitude variation is seen in cv1 (inset). The IC neuron is inhibited during cardiac sac activity; hence cv2 shows a large relaxation followed by a sustained increase in phasic amplitude. cv2 shows very prominent gastric mill-timed variations in contraction amplitude (inset).

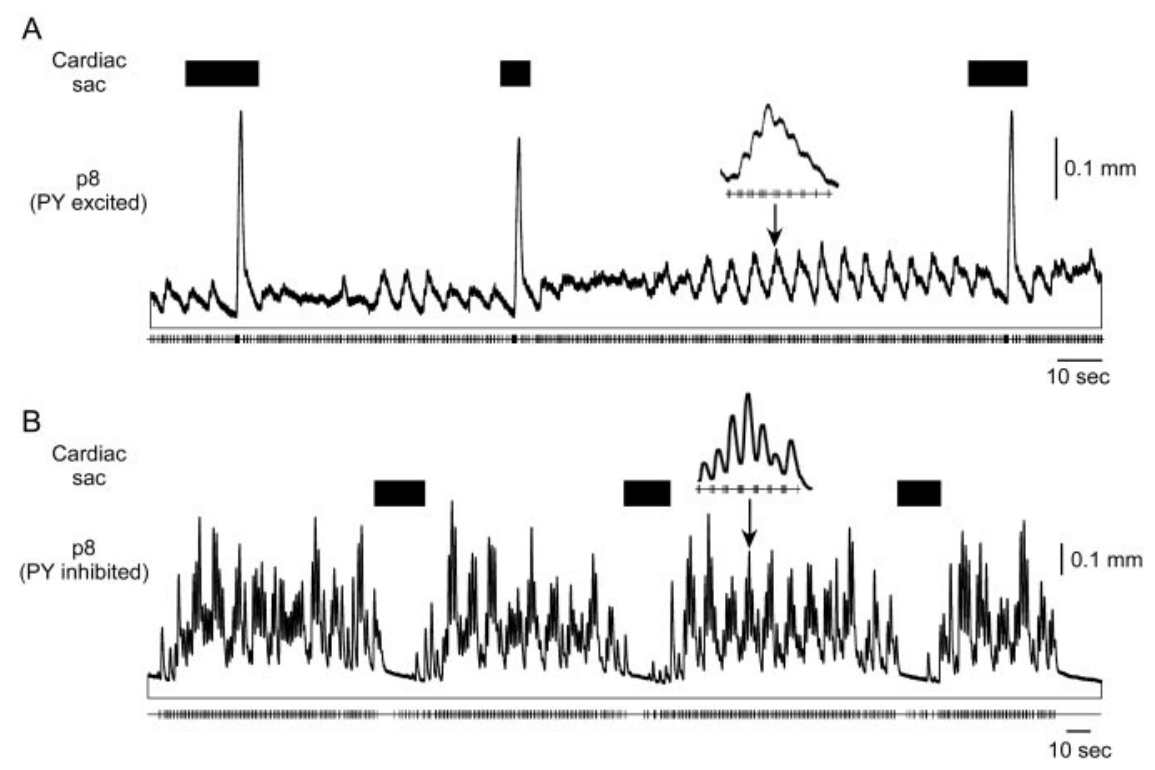

Figure 8. Two responses of $\mathrm{p} 8$ (PY) when the cardiac sac (black rectangles) and gastric mill networks are active. $A$, When stimulated by a PY neuron excited during cardiac sac activity, the muscle shows a very large contraction toward the end of cardiac sac activity. $B$, Stimulation with a PY neuron inhibited by cardiac sac activity causes almost full muscle relaxation during the cardiac sac burst. In both cases gastric mill-timed variations (insets) in amplitude are apparent.

of the total contraction amplitude was tonic. The phasic contraction amplitudes of the muscle were large when driven by the neurons in the middle and bottom preparations but small when driven by the neuron in the top preparation. Similar differences in quantitative response were seen during the cardiac sac burst (presumably because of differences in the firing frequency or duration of the inputs active during cardiac sac bursts that alter PY neuron activity). In the top and bottom panels the PY neuron inhibition lasted the duration of the cardiac sac burst, whereas in the middle panel the PY neuron began firing before the cardiac sac burst was over. 


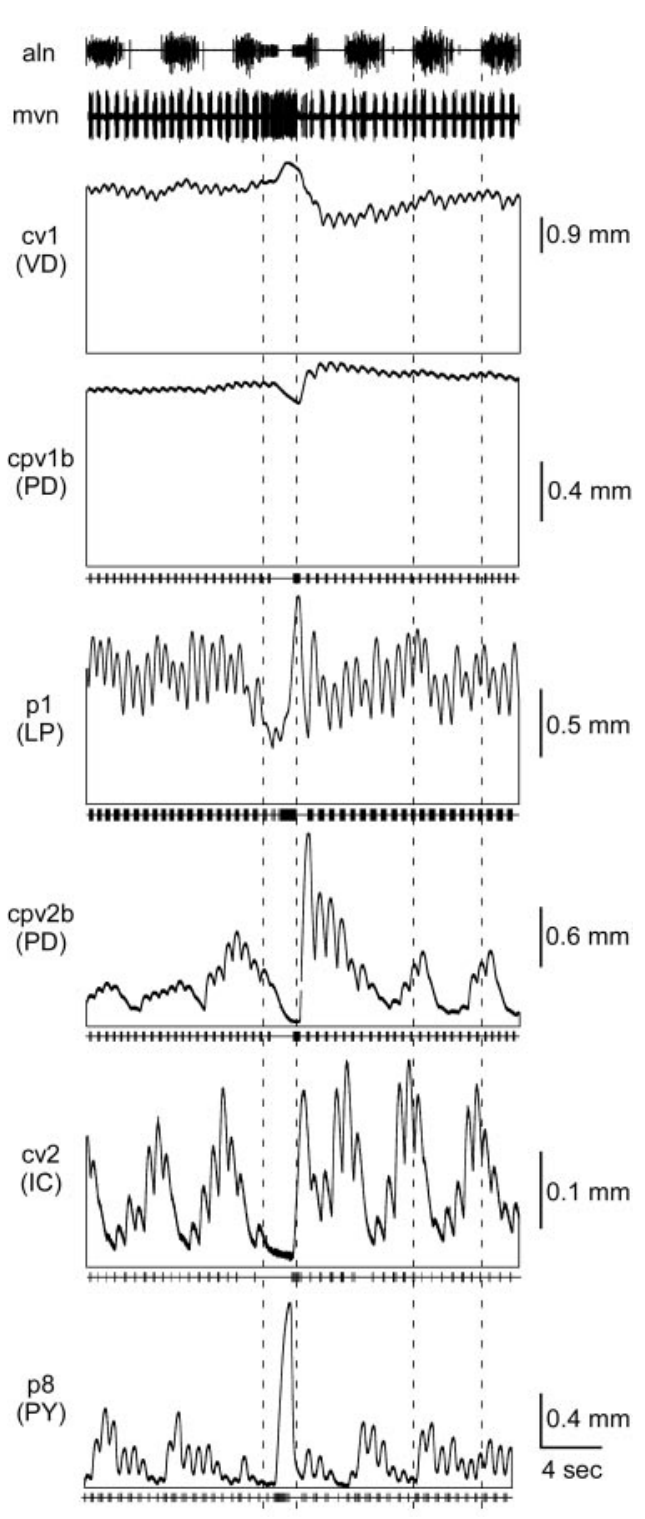

Figure 9. Summary of each pyloric muscle type. Dashed lines show cardiac sac (mvn) burst duration and gastric mill (aln) cycle period. The cv1 (VD), cpv1b (PD), and p1 (LP) muscles show a large tonic contraction that persists throughout gastric mill and cardiac sac activity. cv1 shows no gastric mill-timed variations and an increased amplitude during and sustained decrease after cardiac sac activity. cpv1b shows very small gastric mill-timed variations and a small increase in amplitude before, a decrease during, and a large sustained increase after cardiac sac activity. $p 1$ shows little gastric mill-timed variation in this experiment and a decrease in amplitude during cardiac sac activity. cpv2b (PD) shows gastric mill-timed variations in amplitude and an increase before, a decrease during, and a large increase after cardiac sac activity. cV2 (IC) shows large gastric mill variation and a large relaxation during cardiac sac activity. p8 (PY) shows large gastric mill variation and, because in this experiment a PY neuron excited during cardiac sac activity was used to stimulate the muscle, a large contraction during cardiac sac activity.

This combination of varying muscle response to identical input, and varying neural activity across preparations, made averaging our data impossible. We therefore were forced to show here individual muscle responses to individual examples of neural activity. The data shown in Figure 10, in which the qualitative responses of multiple muscles to the same neural input (left column) and of an individual muscle to multiple neural inputs (right column) were similar, are consistent with this being a valid presentation of the data. Further support is provided by an ex- amination of our entire database, which consists of 9 p1 (LP), 8 p8 (PY), 6 cv2 (IC), 4 cpv1b (PD), 6 cpv2b (PD), and 5 cv1 (VD) muscles and 14 LP neuron, 10 PY neuron, 11 IC neuron, 9 PD neuron, and 12 VD neuron recordings. Not every muscle was stimulated with every neuron recording, but the database contains thousands of gastric mill cycles and $>30$ cardiac sac bursts. Muscle responses qualitatively different from those shown in Figures 5-10 have been observed in none of those cases.

\section{Discussion}

Although the pyloric network is one of the best understood neural networks, the behavior it produces is little understood. The data presented here suggest that the network produces, as a result of the modifications in its activity induced by the gastric mill and cardiac sac networks and the integrative properties of the pyloric muscles, a complex combination of the activities of all three neural networks although only one (the pyloric) innervates them. An important caveat, however, is that in this work muscle loading was constant. The pyloric ossicles are interconnected to form (approximately) a box; thus in the real case resistance to movement around a pyloric "joint" may depend on the length and tension of muscles around other joints (unlike, for instance, limbs, in which shoulder muscle contraction does not alter elbow resistance to movement). A similar concern exists with respect to muscle length in that contraction of some pyloric muscles could alter the length of other pyloric muscles. Our experimental conditions were designed to achieve maximum muscle shortening consistent with full relaxation after a single burst stimulus (see Methods and Materials); thus these joint resistance and muscle length concerns, if true, would result in decreased muscle shortening as compared with the data presented here. Although these concerns cannot be ruled out, the large amplitude of the tonic and gastric mill and cardiac sac-timed contractions and their presence in most pyloric muscles suggest that at least some of these observations likely apply to the in vivo situation.

\section{Relevance to pyloric function}

In the absence of measurements of pyloric muscle force production (as opposed to shortening, as was measured here) and a mechanical model of the pylorus, detailed prediction of pyloric movements is impossible. However, the present data are consistent with three predictions about pyloric function. First, the large tonic contractions produced by some neural input patterns (see below) suggest that the pylorus is sometimes primarily a rigid structure (simultaneous sustained contraction of all pyloric muscles would be expected to stiffen pyloric joints) with only smallamplitude pyloric-timed rhythmic contractions. Second, between cardiac sac bursts many muscles often contract primarily in gastric mill time (Figs. $8 A, \mathrm{p} 8 ; 9$, cpv2b, cv2, p8). For the $\mathrm{p} 8$ muscle, which is located far from the junction of the gastric mill and pylorus, the function of this gastric mill-timed activity is unclear. However, cv2 and cpv2b likely control the movements of the cardiopyloric valve (Maynard and Dando, 1974) through which food moves from the gastric mill to the pylorus. Contraction of these muscles in gastric mill time thus could result in food being moved into the pylorus each gastric mill cycle. Third, the contraction of all pyloric muscles show very large changes, and gastric mill neural output is "disrupted and reset" (Selverston et al., 1976) with every cardiac sac burst. The cardiac sac is a food storage area anterior to the gastric mill in which food is exposed to digestive juices. Cardiac sac constrictions are elicited by a gastric mill neuron and thus occur once each gastric mill cycle (Selverston et al., 1976). The much more infrequent cardiac 

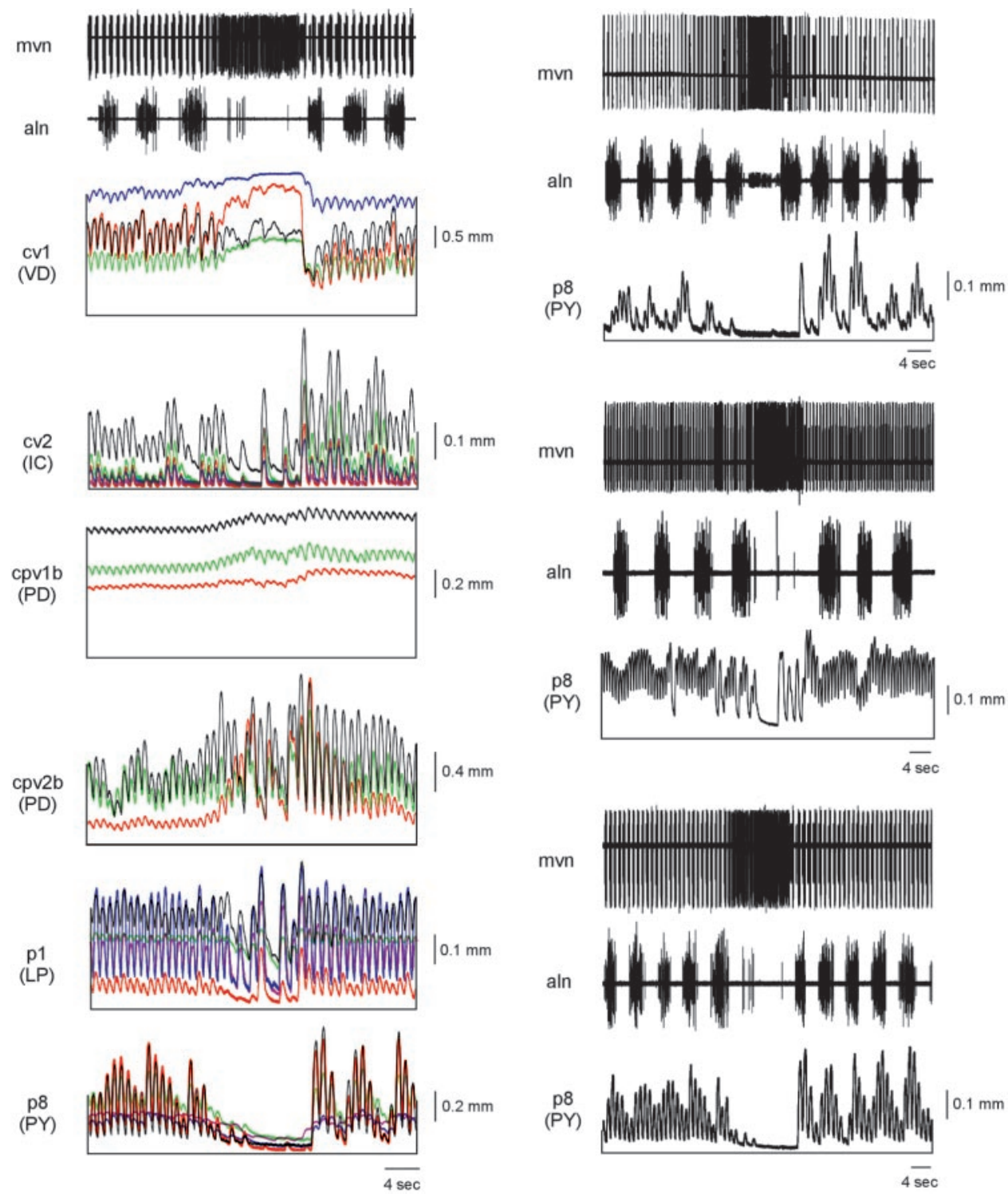

Figure 10. Variation in muscle response to identical driving input and in neuron activity across preparations prevents averaging of muscle response to gastric mill (aln) and cardiac sac (mvn) timed variations in pyloric neuron activity. Left, Five cv1 (VD), five cv2 (IC), three cpv1b (PD), three cpv2b (PD), five p1 (LP), and five p8 (PY) muscles driven by identical neural input containing six gastric mill bursts (aln) and one cardiac sac burst (mvn). Note that in all cases the muscle responses are qualitatively similar but quantitatively different. Right, 0 ne p 8 (PY) muscle driven by three different neural preparations again containing multiple gastric mill cycles (aln) and one cardiac sac burst (mvn). Note again the qualitative similarity of p8 contractions but quantitative differences caused by differences in the PY neuron activity in the three preparations.

sac network bursts, alternatively, dilate the cardiac sac, which would be expected, in the closed fluid-filled lobster stomach, to suck gastric mill and pyloric contents anteriorly. An attractive speculation is thus that the concerted changes in gastric mill and pyloric activity observed during cardiac sac bursts function to redistribute food from these regions toward the cardiac sac.

Taken together, these observations suggest the following hypotheses for pyloric function. Depending on stomach contents, the pylorus varies from being a relatively rigid structure with only small pyloric-timed contractions to being a more flexible organ with large ones. The gastric mill-timed variations in pyloric muscle contraction facilitate passage of chewed food from the gastric mill to the pylorus (similar to how the gastric mill-timed cardiac sac constrictions presumably help to move food from the cardiac sac to the gastric mill). The dilation of the cardiac sac induced by cardiac sac network bursts alternatively would move food from the pylorus to the gastric mill and from the gastric mill to the cardiac sac. This would remove food that had been processed insufficiently by the more anterior regions from the more posterior (a particularly important consideration for the pylorus, because insufficiently chewed but still usable food otherwise must be excreted wastefully) and thus allow stomach contents to be exposed repeatedly to digestive juices (cardiac sac), to be chewed (gastric mill), and to be filtered (pylorus).

\section{Relevance to stomatogastric nervous system evolution and network/cellular properties}

The stomatogastric neural networks, their sensory and modulatory inputs, and the pyloric musculature presumably coevolved to produce the complex intermingled motor output described here. Many aspects of pyloric network neurons and their interconnections thus may exist particularly to support, or to take advantage of, the integrative properties of the pyloric muscles. As 

Duty cycle 0.26

$\mathrm{p} 1$
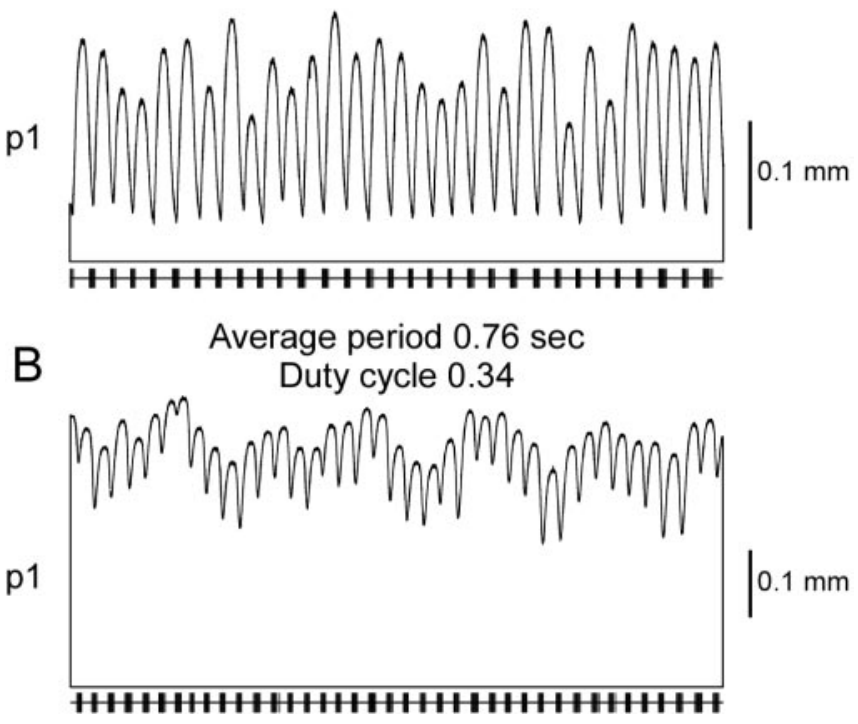

Figure 11. Muscle tonic contraction amplitude depends on cycle period and duty cycle. The same 11 muscle was stimulated with neural recordings with different cycle periods and duty cycles. The amount of tonic contraction depends on how much time the muscle has to relax between bursts (i.e., interburst interval). With a longer cycle period and shorter duty cycle $(A)$ the muscle has more time to relax; therefore, the tonic contraction is smaller than for a shorter cycle period and longer duty cycle $(B)$.

such, it may be impossible to understand the functional basis of certain aspects of the pyloric network (e.g., "why" a given network synapse exists) without a complete understanding of the motor pattern the network produces.

\section{Relevance to pyloric network modulation}

Application of neuromodulatory substances or stimulation of modulatory input pathways induces the pyloric network to produce neural outputs with different cycle periods and neuron duty cycles (burst duration divided by cycle period) (Harris-Warrick and Marder, 1991). The functional consequences of these changes have been unknown but, absent other information, would be expected to produce motor patterns with different cycle periods and muscle contraction duty cycles. The interburst temporal summation and tonic muscle contraction observed in pyloric muscles suggest a different consequence of these changes. Figure 11 shows the activity of the same p1 muscle to neural inputs with different cycle periods and duty cycles. The most salient change the different inputs induced was a shift in muscle activity from being primarily rhythmic in pyloric time (Fig. 11 A) to being mainly tonic with a much reduced rhythmic component (Fig. $11 B$ ). As such, a major consequence of modulator-induced changes in pyloric network output may be to regulate the phasic/ tonic ratio in the contractions the muscles produce.

\section{Relevance to other well studied small systems}

Slow muscles are present in a number of invertebrate systems (Atwood, 1973; Mason and Kristan, 1982; Hall and Lloyd, 1990; McPherson and Blankenship, 1992; Weiss et al., 1992), which suggests that consequences similar to those described here could be present in them as well. In particular, both variable amounts of tonic contraction because of interburst temporal summation and extraction of low-frequency modulation of motor neuron firing could occur. The former of these consequences often has been considered contrary to function, but two of these instances involve animals without hard body wall structures (leech, Aplysia). In these cases tonic contraction could be advantageous by producing muscle stiffness and thus allowing muscles to serve as structural elements. On a more general note, as here, it is likely that in these systems as well a full understanding of why the neural networks innervating these muscles evolved their particular cellular properties and synaptic connectivities, and the functional consequences of sensory and modulatory input to the networks, cannot be understood without taking into consideration the properties of the muscles that the networks innervate.

\section{Spike trains are not enough to predict behavior}

Brezina and colleagues (Brezina and Weiss, 2000; Brezina et al., 2000) have performed detailed theoretical analyses of the consequences of nonlinear neuromuscular transforms and the difficulties they cause in interpreting the functional consequences of motor neuron spike trains. The experimental data presented here are in full concord with their analysis. Particularly important in this regard are the interburst temporal summation and tonic contraction of many pyloric muscles and the very strong facilitation observed in the cpv2b muscle (Fig. 6). Each of these has dramatic functional consequences. The former transforms rhythmic neural input into tonic output, allows extraction of low-frequency signals, and makes changes in motor neuron cycle period and duty cycle (over certain ranges) primarily to alter muscle tonic/ phasic contraction ratio. The second can result in muscles almost exclusively expressing the activities of neural networks that do not innervate them. Neither of these consequences could have been predicted without knowing the integrative properties of the muscles in question. Particularly given the presence of slow muscles in other "model" systems, the theoretical work of Brezina et al. (2000) and the experimental work presented here emphasize the necessity of describing muscle properties to understand how nervous systems generate behavior.

\section{References}

Atwood HL (1973) An attempt to account for the diversity of crustacean muscles. Am Zool 13:357-378.

Brezina V, Weiss KR (2000) The neuromuscular transform constrains the production of functional rhythmic behaviors. J Neurophysiol 83:232-259.

Brezina V, Orekhova IV, Weiss KR (2000) The neuromuscular transform: the dynamic non-linear link between motor neuron firing patterns and muscle contraction in rhythmic behaviors. J Neurophysiol 83:207-231.

Carrier DR (1989) Ventilatory action of the hypaxial muscles of the lizard, Iguana iguana: a function of slow muscle. J Exp Biol 143:435-457.

Claiborne BJ, Selverston AI (1984) Histamine as a neurotransmitter in the stomatogastric nervous system of the spiny lobster. J Neurosci 4:708-721.

Ellis TA, Donath AS, Morris LG, Thuma JB, Hooper SL (1996) Motor expression of a lateral pyloric constrictor muscle. Soc Neurosci Abstr 22:131.

Hall JD, Lloyd PE (1990) Involvement of pedal peptide in locomotion in Aplysia: modulation of foot muscle contractions. J Neurobiol 21:858-868.

Harness PI (1998) Prediction of spiny lobster stomatogastric muscle contractions in response to physiologically relevant constant duty cycle rhythmic neural input. Senior thesis, Ohio University.

Harness PI, Morris LG, Hooper SL (1998) Intrinsic pyloric muscle output predicted from constant duty cycle rhythmic neural input. Soc Neurosci Abstr 24:1891.

Harris-Warrick RM, Marder E (1991) Modulation of neural networks for behavior. Annu Rev Neurosci 14:39-57.

Hetherington TE, Lombard RE (1983) Electromyography of the opercularis muscle of Rana catesbeiana: an amphibian tonic muscle. J Morphol 175:17-26.

Hoover NJ, Weaver AL, Harness PI, Hooper SL (2002) Combinatorial and cross-fiber averaging transform muscle electrical responses with a large 
stochastic component into deterministic contractions. J Neurosci 22:1895-1904.

Jorge-Rivera JC, Marder E (1996) TNRNFLRFamide and SDRNFLRFamide modulate muscles of the stomatogastric system of the crab Cancer borealis. J Comp Physiol [A] 179:741-751.

Koehnle TJ, Morris LG, Thuma JB, Hooper SL (1997) Motor activity of the pyloric and cardiac sac innervated cv1 muscle. Soc Neurosci Abstr 23:477.

Marder E, Calabrese RL (1996) Principles of rhythmic motor pattern generation. Physiol Rev 76:687-717.

Mason A, Kristan WBJ (1982) Neuronal excitation, inhibition, and modulation of leech longitudinal muscle. J Comp Physiol [A] 146:527-536.

Maynard DM, Dando MR (1974) The structure of the stomatogastric neuromuscular system in Callinectes sapidus, Homarus americanus, and Panulirus argus (Decapoda Crustacea). Philos Trans R Soc Lond B Biol Sci 268:161-220.

McPherson DR, Blankenship JE (1992) Neuronal modulation of foot and body-wall contractions in Aplysia californica. J Neurophysiol 67:23-28.

Meyrand P, Marder E (1991) Matching neural and muscle oscillators: control by FMRFamide-like peptides. J Neurosci 11:1150-1161.

Meyrand P, Moulins M (1986) Myogenic oscillatory activity in the pyloric rhythmic motor system of Crustacea. J Comp Physiol [A] 158:489-503.

Morris LG (1998) Responses of a slow muscle to changing neuronal input in the stomatogastric system of the spiny lobster (Panulirus interruptus). $\mathrm{PhD}$ thesis, Ohio University.

Morris LG, Hooper SL (1996) The pyloric dilator muscles show very different contraction amplitude, facilitation, and summation. Soc Neurosci Abstr 22:131.

Morris LG, Hooper SL (1997) Muscle response to changing neuronal input in the lobster (Panulirus interruptus) stomatogastric system: spike number- versus spike frequency-dependent domains. J Neurosci 17:5956-5971.

Morris LG, Hooper SL (1998) Muscle response to changing neuronal input in the lobster (Panulirus interruptus) stomatogastric system: slow muscle properties can transform rhythmic input into tonic output. J Neurosci 18:3433-3442.

Morris LG, Hooper SL (2001) Mechanisms underlying stabilization of temporally summated muscle contractions in the lobster (Panulirus) pyloric system. J Neurophysiol 85:254-268.

Morris LG, Thuma J, Hooper SL (1999) Pyloric muscles can express gastric and cardiac sac contraction patterns despite pyloric-timed motor neuron firing. Soc Neurosci Abstr 25:1642.

Morris LG, Thuma JB, Hooper SL (2000) Muscles express motor patterns of non-innervating neural networks by filtering broad-band input. Nat Neurosci 3:245-250.

Mulloney B (1977) Organization of the stomatogastric ganglion of the spiny lobster. V. Coordination of the gastric and pyloric systems. J Comp Physiol [A] 122:227-240.

Russell DF, Hartline DK (1981) A multiaction synapse evoking both EPSPs and enhancement of endogenous bursting. Brain Res 223:19-38.

Selverston AI, Russell DF, Miller JP, King DG (1976) The stomatogastric nervous system: structure and function of a small neural network. Prog Neurobiol 7:215-290.

Sigvardt KA, Mulloney B (1982) Properties of synapses made by IVN command-interneurones in the stomatogastric ganglion of the spiny lobster Panulirus interruptus. J Exp Biol 97:153-168.

Thuma JB, Hooper SL (2002) Quantification of gastric mill network effects on a movement-related parameter of pyloric network output in the lobster. J Neurophysiol 87:2372-2384.

Thuma JB, Hooper SL (2003) Quantification of cardiac sac network effects on a movement-related parameter of pyloric network output in the lobster. J Neurophysiol 89:745-753.

Weiss KR, Brezina V, Cropper EC, Hooper SL, Miller MW, Probst WC, Vilim FS, Kuperfermann I (1992) Peptidergic co-transmission in Aplysia: functional implications for rhythmic behaviors. Experientia 48:456-463. 